\title{
Modular pumps as programmable hydraulic batteries for microfluidic devices
}

\author{
Brian M. Cummins, Rukesh Chinthapatla, Balaji Lenin, Frances S. Ligler \& Glenn M. Walker
}

Simple fluid pumps have been developed to improve microfluidic device portability, but they cannot be easily programmed, produce repeatable pumping performance, or generate complex flow profiles - key requirements for increasing the functionality of portable microdevices. We present a detachable, paper-based, "hydraulic battery" that can be connected to the outlet of a microfluidic channel to pump fluid at varying flow rates over time, including step changes, ramping flows, and oscillating flows.

Keywords: Paper Microfluidics; Capillary Pump; Microfluidic; Lateral Flow; Microfluidic Pump; Point of Care.

\section{INNOVATION}

The hydraulic battery demonstrated here is a novel, paper-based pump that uses paper geometry to achieve preprogrammed flow rates and durations. Pumps are designed by choosing the dimensions of the resistive neck and absorption region, which control the pumping flow rate and volume, respectively. Lamination encapsulates the paper and minimizes the evaporation that plagues other paper microfluidics, improving pumping reproducibility. The pumps are fabricated using a simple, scaleable procedure: here, chromatography paper is laminated and then cut to the desired shape with a laser cutter, although a variety of porous materials can be used. Multiple pumps can be stacked with or without dissolvable delays to create complex flow profiles. Using hydraulic batteries, users can generate varying flow rate profiles in microfluidic devices, including both increasing and decreasing step changes, ramping flow, and oscillating flow.

\section{NARRATIVE}

\section{Introduction}

Fluid transport in microfluidic devices is usually controlled by pumps that require external power and cumbersome fluidic connections, limiting the portability of the system ${ }^{1}$. On-chip pumps exist, but achieving a desired flow rate can be an empirical process and the pumps cannot generate complex flow behavior ${ }^{2-7}$. Capillary pumps have been fabricated via photolithography within microfluidic devices to pull fluids through microchannels ${ }^{8-10}$, but their integration within the device prevents exchanging or replacing the pumps. To lower the cost and fabrication time of capillary pumps, several groups have used paper connected to the distal end of a microfluidic channel to pull fluid ${ }^{11-14}$. These paper pumps use simple geometric shapes to generate a single flow rate but they only work on channels with relatively low fluidic resistances and are built into the analytical test substrate. Wang et al. demonstrated a clever approach for programming detachable paper-based pumps to pump at progressively slower flow rates ${ }^{15}$. However, this strategy cannot be used to create more complex flow profiles, such as increasing flow rates, oscillating flow, or delayed-onset flow.

Paper devices have been enhanced by improvements such as delays and evaporation barriers, among others. Lamination strategies for paper-based devices include the use of printer toner ${ }^{16}$, tape $\mathrm{e}^{17}$ and lamination with plastic sheets ${ }^{18,19}$. Dissolvable sugar barriers have been implemented to create fluid delays ${ }^{20,21}$ and to create timed shutoff valves ${ }^{22}$. Thin films of polyvinyl alcohol (PVA) have been used to create fluid delays ${ }^{23,24}$ in polymer devices and meter precise volumes of liquid samples in paper ${ }^{25}$, but they have not been used as delays to control fluid flow within paper.

In this communication, we extend previous work on shaped paper, dissolvable barriers, and lamination and combine them in a novel way to create a paper-based pump capable of complex pumping operations. Each pump comprises one region that controls the volumetric flow rate independently of the inlet pressure and fluidic resistance of the upstream microfluidic channels and another region that controls the volumetric capacity of the pump. The geometry of these two regions encodes the unique flow-rate profiles (volumetric flow rate vs. time) for a given pump. We have demonstrated preprogrammed paper pumps that produce a range of flow rates $(0.64-5.5 \mu \mathrm{L} / \mathrm{min})$ and pumping volumes $(8-528 \mu \mathrm{L})$. Multiple pumps were stacked to increase flow rates or to generate complex flow rate profiles within attached microfluidic channels.

\section{Methods}

Because the pumps actuate microfluidic devices by pumping fluid through them, we call the pumps "hydraulic batteries" as they are analogous to the disposable, miniature electrical batteries that power portable electronic devices. Because of their similarity to alkaline batteries, which are rated in volts and amp-hours, we rate hydraulic batteries in terms of their flow

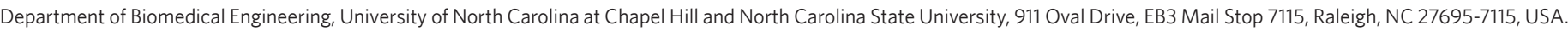
Correspondence to: F.S.L. (fsligler@ncsu.edu) or G.M.W. (gmwalker@ncsu.edu)

Received 16 September 2016; accepted 25 January 2017; published online 24 February 2017; doi:10.1142/S2339547817200011

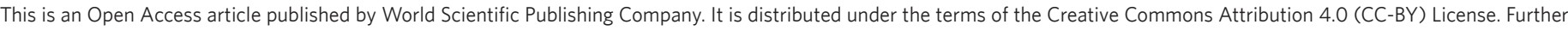
distribution of this work is permitted, provided the original work is properly cited. 
rate and pumping duration. For example, a hydraulic battery designed to pump $32 \mu \mathrm{L}$ of water $(\mathrm{w})$ at $1.5 \mu \mathrm{l} / \mathrm{min}$ would be designated as a $32 \mathrm{w} 1.5$ battery (Supplementary Table 1).

The hydraulic battery is divided into three regions: (1) an inlet region that is connected to the microfluidic device, (2) a porous "flow-rate control" region that has a fluidic resistance greater than the microchannel to which it is connected, and (3) an absorbent region that controls the volume of liquid imbibed (Fig. 1a and Supplementary Fig. 1). The hydraulic battery is affixed to the outlet of a microchannel and, once wetted, controls the flow rate and duration of fluid transport (Fig. 1b). The flow-rate control region should have a hydraulic resistance $\sim 10$ times greater than the resistance of the upstream microfluidic device to minimize variations in flow caused by the attached microdevice. In the devices tested here, the resistance of the flow-rate control region varies slightly between designs but was $\sim 1 \times 10^{13} \mathrm{~kg} /\left(\mathrm{m}^{4} \mathrm{sec}\right)$ compared to 7.8 $\times 10^{11} \mathrm{~kg} /\left(\mathrm{m}^{4} \mathrm{sec}\right)$ for the microbore tubing attached for the purpose of
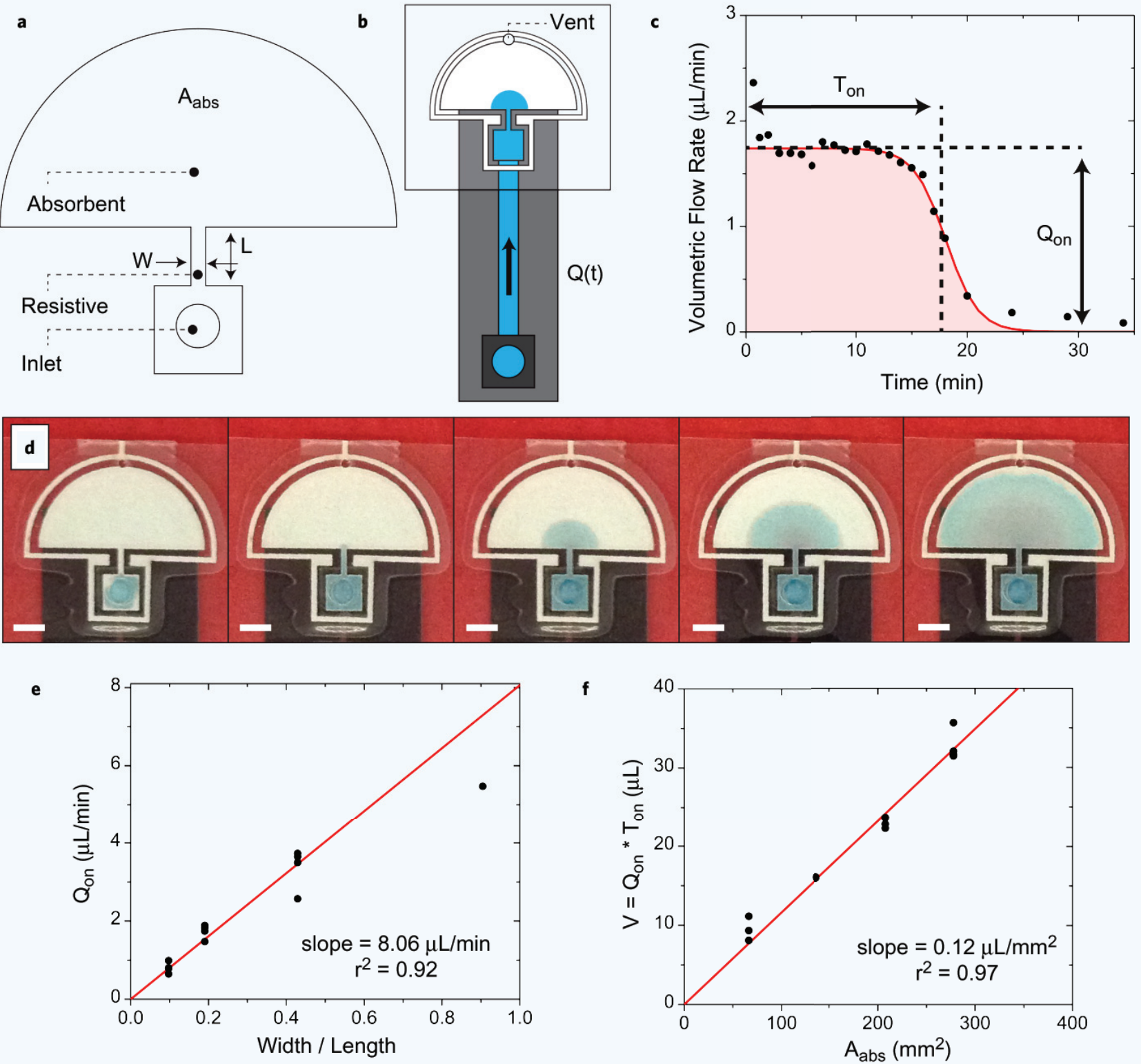

Figure 1 Basic hydraulic battery. (a) Schematic of a basic hydraulic battery showing the inlet region, resistive region with width, $W$, and length, $L$, and the absorbent region with area, $A_{\text {abs. }}$. Fluid is absorbed through the inlet port. (b) Schematic of a basic hydraulic battery attached to a microfluidic device. The absorption into the porous material determines the volumetric flow rate, $Q(t)$ through the device. (c) Representative flow-rate profile (volumetric flow rate vs. time) of a basic hydraulic battery with $W / L=0.19$ and $A_{a b s}=277 \mathrm{~mm}^{2}$. From the best fit, this specific battery is rated to have a measured $Q_{o n}=1.7 \mu \mathrm{L} / \mathrm{min}$ and a measured $T_{\text {on }}=18.1 \mathrm{~min}$. The shaded area under the curve is equal to the volume absorbed, $V=$ $\mathrm{Q}_{\mathrm{on}} \cdot T_{\text {on }}(31 \mu \mathrm{L})$. (d) Once the fluid in the microchannel contacts the hydraulic battery, fluid is pulled into the pump. Images are taken at 2 seconds, 15 seconds, 2 minutes, 6 minutes, and 20 minutes after the fluidic connection is made. Scale bar is $5 \mathrm{~mm}$. (e) The rated volumetric flow rate, $Q_{o n}$ is a function of the $W / L$ ratio of the resistive region. Black dots represent measured flow rates from hydraulic batteries with a range of $W / L$ ratios. (f) The rated volume, $V$, of a hydraulic battery is a function of the area of the absorbent region, $A_{\text {abs. }}$. Black dots represent measured volume absorbed for different $A_{\text {abs }}$. In (e) and (f), the lines were best fits of experimental data points (Supplementary Information). 
measuring the flow rates over extended distances. Typical microchannel resistances were $1 \times 10^{9} \mathrm{~kg} /\left(\mathrm{m}^{4} \mathrm{sec}\right)$.

A wide range of porous materials can be used as the pumping substrate as long as the pores within the matrix are interconnected and the fluid wets the material. For this demonstration, hydraulic batteries were fabricated by shaping laminated Whatman \# 1 chromatography paper with a laser cutter and laminating the cut-out to minimize evaporation (details in Supplementary Information (SI), Supplementary Fig. 2, Supplementary Table 2). Holes in the plastic films at opposite ends of the pump provided a port for the porous material to be connected to the microchannel and a vent for release of air displaced by incoming fluid, respectively.

Hydraulic batteries were attached to microfluidic channels or microbore tubing primed with water containing blue dye to measure performance. Once fluid contacted the pump, displacement through the channel or tubing was visually tracked to determine the volumetric flow rate, $Q$, vs. time, $t$, profile of the pump (Supplementary Fig. 3). This experimental flow-rate profile was fit to Equation (1) using a nonlinear least squares method to determine ratings for each battery's pumping performance (SI, Supplementary Table 3). The extracted values for $Q_{\mathrm{on}}$ and $T_{\mathrm{on}}$ are the steady-state flow rate and the duration of pumping, respectively. The total volume absorbed by the pump, $V$, is equal to $Q_{\text {on }} \bullet T_{\text {on }}$. The variable $c$ is a time constant that accounts for the sloped decay of the flow rate. Figure 1c shows the fit for a hydraulic battery with $Q_{\text {on }}=1.7 \mu \mathrm{L} / \mathrm{min}$ and $\mathrm{a} T_{\text {on }}=18.1 \mathrm{~min}$.

$$
Q(t)=\frac{Q_{\text {on }}}{1+e^{\left(t-T_{\text {on }}\right) / c}}
$$

\section{Results/Discussion}

For a single, continuous porous material, the volumetric flow rate and volume of fluid absorbed can be adjusted by changing the geometries of the resistive and absorbent regions, respectively (Fig. 1e,f). The steady-state flow rate, $Q_{\mathrm{on}}$, is proportional to the width-to-length ratio of the resistive region, $W / L$, and the total absorbed volume, $V$, is proportional to the area of the absorbent region, $A_{\mathrm{abs}}$. The slopes of the lines in Fig. 1e and Fig. 1f will vary for different fluid and porous material combinations.

Pumps were designed to demonstrate the effect of geometry on pumping performance. Varying $W / L$ while maintaining a constant pumping volume $V$ (Fig. 2a and Supplementary Movie 1) showed that a larger $W / L$ ratio $(0.90)$ yields a faster flow rate (5.5 $\mu \mathrm{L} / \mathrm{min})$ for a shorter from experimental data. duration $(5.9 \mathrm{~min})$, while a smaller $W / L$ ratio $(0.10)$ creates a slower flow rate $(0.8 \mu \mathrm{L} / \mathrm{min})$ for a longer duration $(44.2 \mathrm{~min})$. Short duration pumps showed greater flow-rate variability during $Q_{\mathrm{on}}$ while slower pumps appeared more stable. This variability can be partly explained by less accurate measurement of the lagging front moving through the microfluidic channel at the faster rates.

Figure $\mathbf{2 b}$ and Supplementary Movie 2 show the effect of changing $A_{\text {abs }}$ while maintaining $W / L=0.10\left(Q_{\mathrm{on}}=0.8 \mu \mathrm{L} / \mathrm{min}\right)$. These batteries were designed to absorb different volumes $(8,16,24$, and $32 \mu \mathrm{L})$ and were expected to pump for different times (9.8, 20.1, 30.8, and $41.2 \mathrm{~min}$ ); measured $T_{\text {on }}$ values were 11.2, 25.0, 30.0 and 44.2 minutes, respectively. A $455 \mathrm{w} 0.8$ battery pumped fluid for $676 \mathrm{~min}$ ( $>11$ hours) at $0.8 \mu \mathrm{l} / \mathrm{min}$; such a design could be useful for extended pumping applications such microfluidic cell culture.

Multiple hydraulic batteries were stacked to produce complex pumping behavior within a microchannel. Openings on each face of the inlet region were created to allow fluid flow between neighboring pumps via connecting paper disks. After the initial wetting of the inlet region connected to the microchannel, the wetted front advanced vertically through the entire stack through the inlets with low fluidic resistance and then horizontally into the high fluidic resistance regions of the individual batteries (Fig. 3a). The flow rates within each battery added to increase the overall flow rate within the microchannel. Figure $\mathbf{3 b}$ shows the effect of stacking $\mathrm{N}=1, \mathrm{~N}=2$, and $\mathrm{N}=3$ pumps that pumped total volumes of 32,64 , and $96 \mu \mathrm{L}$ at $1.5,3.0$, and $4.5 \mu \mathrm{L} / \mathrm{min}$, respectively. Stacking proved to be an effective strategy to increase the sample volume pulled through an attached microfluidic device without significantly increasing the device footprint.

Stacked pumps were also used to achieve more complex flow-rate profiles. For example, a step down in flow rate, previously demonstrated by Wang et al. ${ }^{15}$, was generated using two pumps with different geometries, as shown in Fig. 3c. The two different geometries absorbed the same
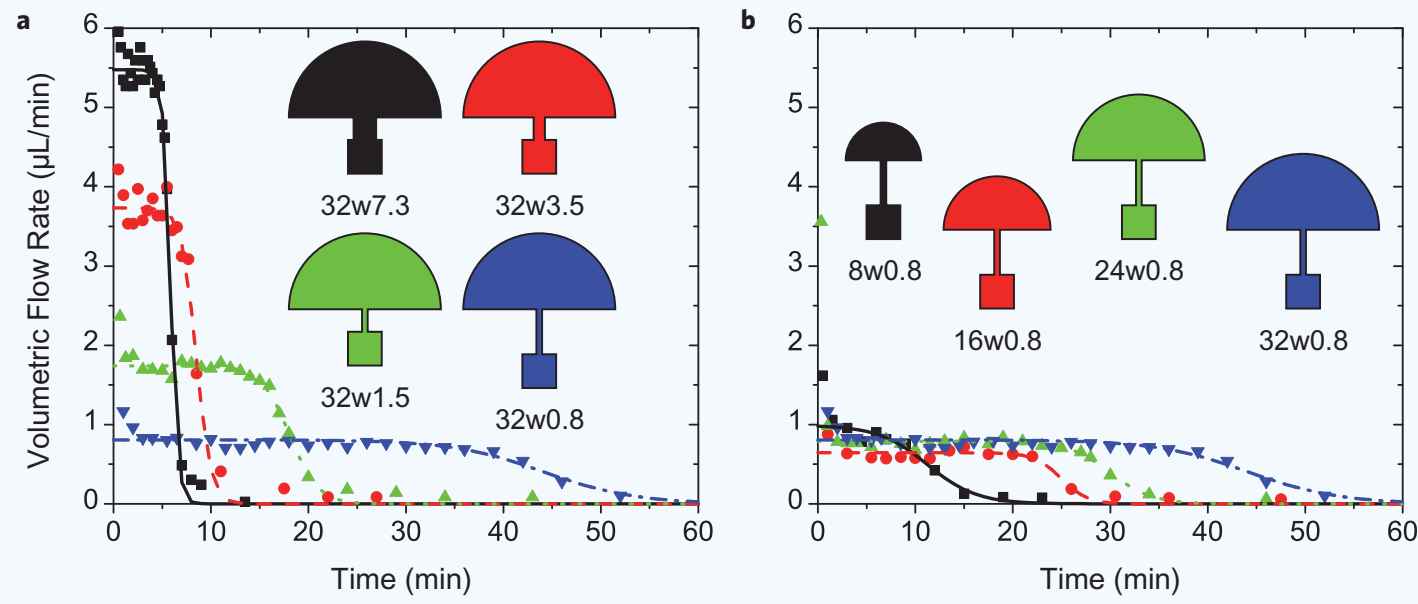

Figure 2 Flow-rate profiles of different hydraulic batteries. (a) Pumps that have the same $A_{\text {abs }}$ but different $W / L$ ratios pump the same volume (area under the curve) at different flow rates. (b) Pumps that have the same $W / L$ ratio but different $A_{\text {abs }}$ pump different volumes at the same flow rate. For each graph, the measured points and best-fit curves are

Supplementary Movie 1 ("This movie displays the performance of a set of hydraulic batteries that have the same absorbent region but different resistive regions. The position of the lagging front of the fluid is indicative of the volumetric flow rate of the battery. The volumetric flow rate is a function of the resistive region. The timer (HH:MM:SS) shows the real time from initial loading") can be viewed at http://www.worldscientific. com/doi/suppl/10.1142/S2339547817200011
Supplementary Movie 2 ("This movie displays the performance of a set of hydraulic batteries that have the same resistive region but different absorbent regions. The position of the lagging front of the fluid is indicative of the volumetric flow rate of the battery. The volumetric flow rate is the same for each hydraulic battery while pumping but the total volume pumped is a function of the absorbent region of the hydraulic battery. The timestamp (HH:MM:SS) is the real time of the movie") can be viewed at http://www. worldscientific.com/doi/suppl/10.1142/S2339547817200011 


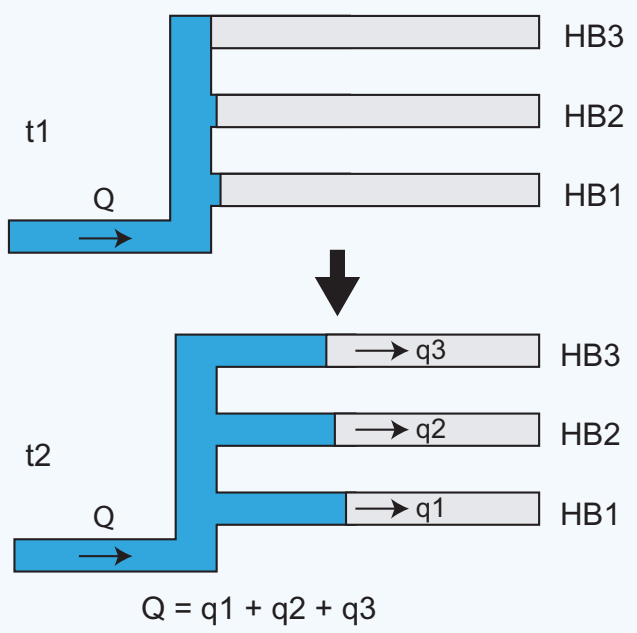

c

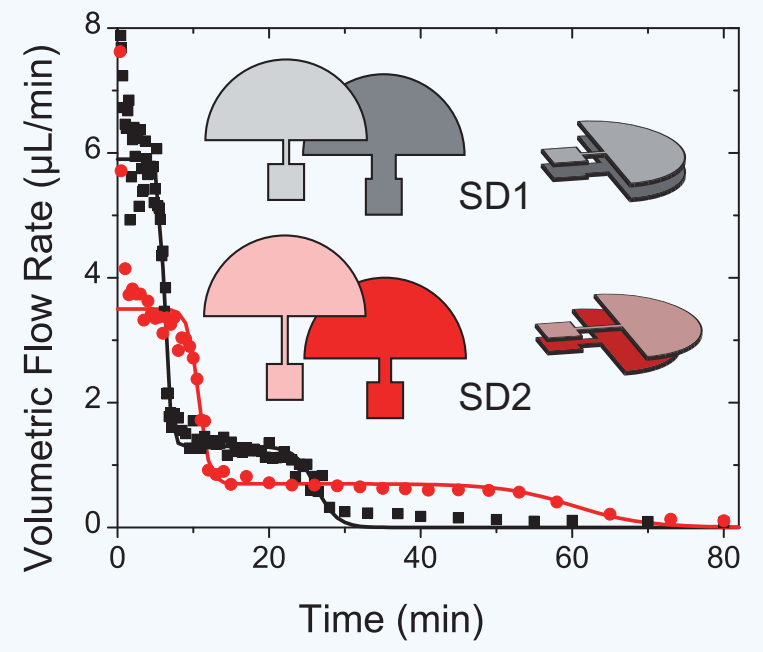

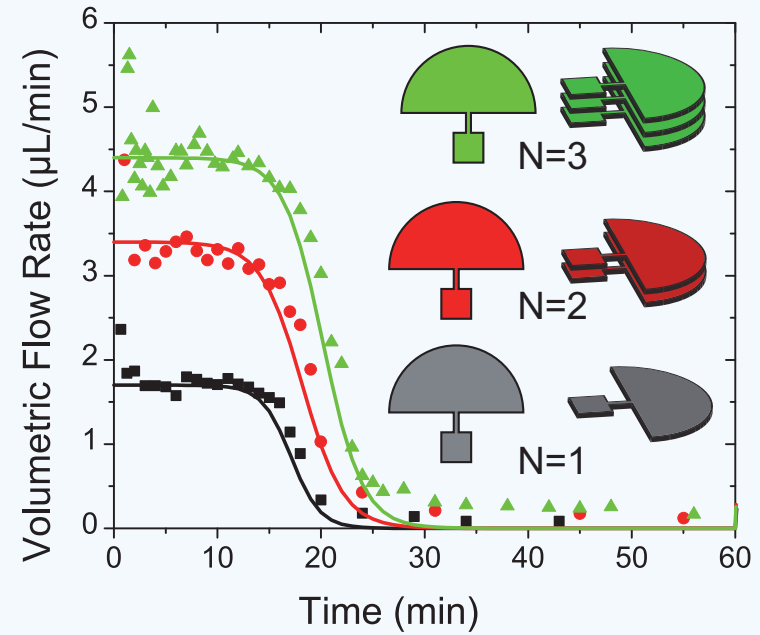

d

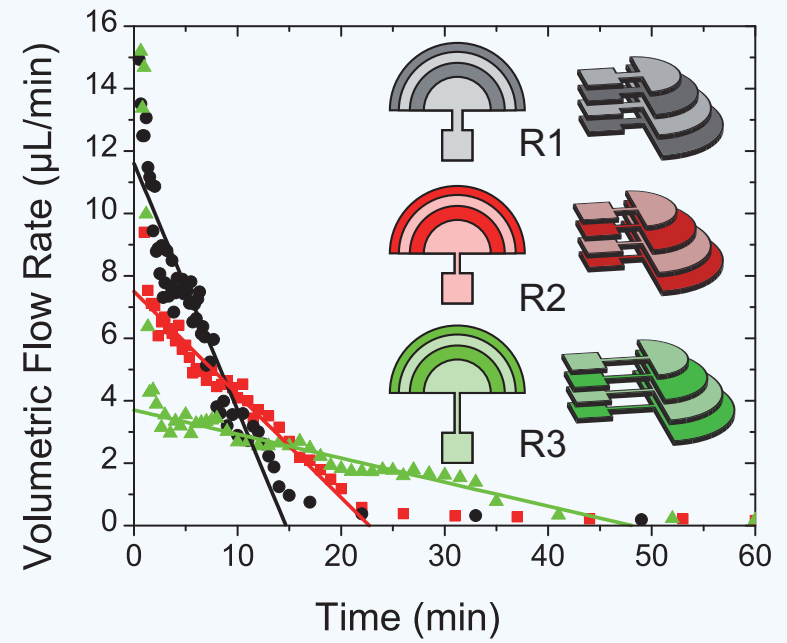

Figure 3 Performance of stacked hydraulic batteries. Stacks can be used to generate more complex flow profiles. (a) Schematic of how a stack of hydraulic batteries ( $H B 1, H B 2, H B 3$ ) absorbs fluid. First, all inlet regions fill at approximately the same time (t1) with minimal absorption into the batteries. Then, the wetted front advances down the resistive regions ( $t 2)$. The flow rate $Q$ through the microfluidic channel is the sum of the flow rates through each pump. (b) Experimental flow rate profiles of stacks $(N=1, N=2$, and $N=3$ ) of identical batteries showing that flow rates can be increased for a given footprint. (c) Step-down flow rate profiles (SD1 and SD2) were achieved experimentally using stacks of two pumps with different resistive regions. (d) Experimental flow-rate profiles of three ramping stacks (R1, R2, and R3), showing that the sloping decreased over time as predicted from combining the performance parameters of the pumps selected. For a given ramping stack, each pump has the same resistive region but different absorbent regions. For each graph, the measured points and best-fit curves are from experimental data.

fluid volumes but over different times and with different step-down flow profiles.

Linearly decreasing flow rates were created with a stack of batteries containing identical resistive regions but absorbent regions of decreasing areas (Fig. 3d). The maximum flow rate, the slope of the ramp, and the resolution of the steps can be controlled by adjusting the number of batteries, the $W / L$ ratio, and the rate of change in $A_{\mathrm{abs}}$ of the different batteries in the stack (SI). Figure 3d shows three ramps that absorb the same volume $(\sim 80 \mu \mathrm{L})$, but with $Q_{\max }=11.6,7.5$, and $3.7 \mu \mathrm{L} / \mathrm{min}$, and $m_{\text {ramp }}=-0.79,-0.33$, and $-0.077 \mu \mathrm{L} / \mathrm{min}^{2}$, respectively. A smoother ramp can be created by increasing the number of pumps and adapting the resistive and absorbent regions.

Even more complex flow-rate profiles were created by introducing dissolvable barriers (i.e., time delays) between batteries in a stack (Fig. 4, Supplementary Fig. 4a, and SI). Figure $4 \mathrm{~b}$ shows representative flow-rate profiles of a hydraulic battery (32w7.3) connected directly to a microfluidic channel and the same battery with a delay inserted between the pump inlet and the microfluidic channel. The battery without a delay immediately pumped fluid while the battery with the delay did not begin pumping for 20 minutes. Figure $4 \mathrm{c}$ and Supplementary Fig. $4 \mathrm{~b}$ show a stack of batteries and delays that created an oscillating flow-rate profile. In this design, the battery (32w0.8) immediately connected to the microfluidic channel pumps for the duration of the experiment, while the next two batteries in the stack pumped only after the delay dissolved. This stack created a flow rate of approximately $1 \mu \mathrm{L} / \mathrm{min}$ from 0-5 min, $6 \mu \mathrm{L} / \mathrm{min}$ from $5-10 \mathrm{~min}, 1 \mu \mathrm{L} / \mathrm{min}$ from $10-15 \mathrm{~min}, 4.5$ $\mu \mathrm{L} / \mathrm{min}$ from $15-23 \mathrm{~min}$, and $1 \mu \mathrm{L} / \mathrm{min}$ from $23-45 \mathrm{~min}$. This experiment shows proof-of-concept that a complex flow rate profile can be built with mix-and-match components and exhibits the potential flexibility and adaptability of hydraulic batteries. 


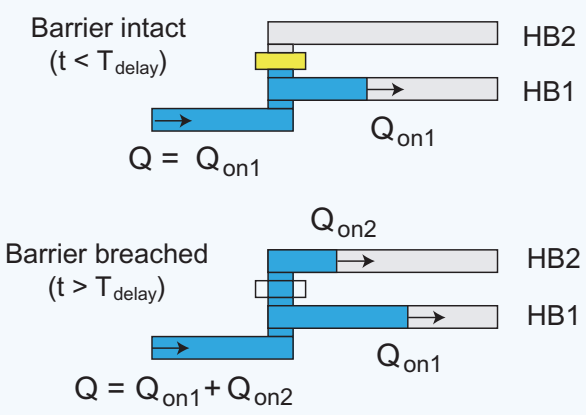

b

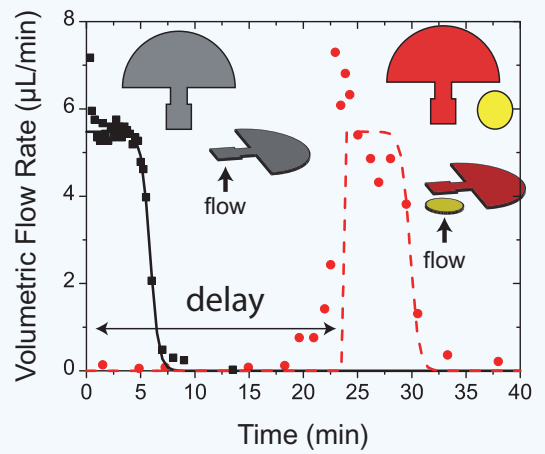

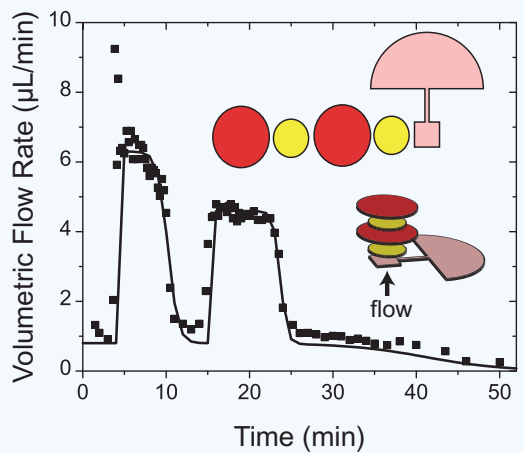

Figure 4 Performance of stacks with time delays. (a) Schematic of the dissolvable time delay. The delay prevents fluid from wicking into downstream batteries until it is breached. The flow rate $Q$ in the attached microchannel is the sum of the flow rates into each pump. (b) Experimental flow rate profile of a 32 w7.3 battery without a delay compared to the profile of the same battery with a $20 \mu \mathrm{m}$ thick (PVA) disk between the microfluidic channel and the inlet, showing the time delay in the volumetric flow rate profile. (c) Oscillating flow rate profile generated by a stack that included multiple delays. The order of the stack was a 32w0.8 battery, $15 \mu \mathrm{m}$ thick PVA delay, circular battery, $15 \mu \mathrm{m}$ thick PVA delay, and circular battery. After each PVA delay broke, the flow rate increased from the $Q_{o n}$ of the $32 w 0.8$ to a higher rate until its downstream circular battery is saturated. The rate then returned to the $Q_{o n}$ of $32 w 0.8$. For each graph, the measured points and best-fit curves are from experimental data.

\section{CONCLUSIONS}

Stackable, self-powered pumps can transport fluid through microfluidic chips with a wide range of pre-defined flow-rate profiles. Dissolvable barriers can be added to further extend the functionality. The material cost of this approach is currently $<\$ 0.07$ USD per battery (Supplementary Table 4), and could be decreased even further using roll-to-roll fabrication methods. The pumps are suitable for a wide variety of applications including diagnostics, microfluidic cell culture, and process control, and the designs can be adapted for a wide variety of fluids, porous materials, and environments. Complex flow profiles could, for instance, accommodate immunoassays with multiple sample/reagent addition, incubation and wash steps. Slow flow/high volume pumps could be used to draw culture fluid through multiple, parallel cell cultures without running tubing through an incubator; pumps could be sequentially removed from stacks for biochemical analysis of the effluents. A collection of pumps with known resistances for a particular fluid could be used for quality control during production of porous materials. Hydraulic batteries have the potential to take more microfluidic devices out of the lab, analogous to the manner in which miniature electric batteries have put computers and phones in our pockets.

\section{ACKNOWLEDGEMENTS}

This work was supported by the Chancellor's Innovation Fund and the Ross Lampe Chair in Biomedical Engineering at North Carolina State University.

\section{REFERENCES}

1. Woias, P. Micropumps - Past, progress and future prospects. Sensor Actuat. B Chem. 105, 28-38 (2005)

2. Marmottant, P. \& Hilgenfeldt, S. A bubble-driven microfluidic transport element for bioengineering. Proc. Natl. Acad. Sci. U.S.A. 101, 9523-9527 (2004).

3. Walker, G.M. \& Beebe, D.J. A passive pumping method for microfluidic devices. Lab Chip 2, 131-134 (2002).

4. Dimov, I.K. et al. Stand-alone self-powered integrated microfluidic blood analysis system (SIMBAS). Lab Chip 11, 845-850 (2011).

5. Begolo, S., Zhukov, D.V., Selck, D.A., Li, L. \& Ismagilov, R.F. The pumping lid: Investigating multi-material 3D printing for equipment-free, programmable generation of positive and negative pressures for microfluidic applications. Lab Chip 14, 4616-4628 (2014).
6. Iwai, K. et al. Finger-powered microfluidic systems using multilayer soft lithography and injection molding processes. Lab Chip 14, 3790-3799 (2014).

7. Xu, K., Begley, M.R. \& Landers, J.P. Simultaneous metering and dispensing of multiple reagents on a passively controlled microdevice solely by finger pressing. Lab Chip 15, 867-876 (2015)

8. Zimmermann, M., Schmid, H., Hunziker, P. \& Delamarche, E. Capillary pumps for autonomous capillary systems. Lab Chip 7, 119-125 (2007).

9. Lillehoj, P.B., Wei, F. \& Ho, C.M. A self-pumping lab-on-a-chip for rapid detection of botulinum toxin. Lab Chip 10, 2265-2270 (2010).

10. Safavieh, R. \& Juncker, D. Capillarics: Pre-programmed, self-powered microfluidic circuits built from capillary elements. Lab Chip 13, 4180-4189 (2013).

11. Xu, Z.R. et al. A microfluidic flow injection system for DNA assay with fluids driven by an on-chip integrated pump based on capillary and evaporation effects. Lab Chip 8, 1658-1663 (2008).

12. Wang, J. et al. A self-powered, one-step chip for rapid, quantitative and multiplexed detection of proteins from pinpricks of whole blood. Lab Chip 10, 3157-3162 (2010).

13. Kokalj, T., Park, Y., Vencelj, M., Jenko, M. \& Lee, L.P. Self-powered imbibing microfluidic pump by liquid encapsulation: SIMPLE. Lab Chip 14, 4329-4333 (2014).

14. Chumo, B., Muluneh, M. \& Issadore, D. Laser micromachined hybrid open/paper microfluidic chips. Biomicrofluidics 7, 064109 (2013).

15. Wang, X., Hagen, J.A. \& Papautsky, I. Paper pump for passive and programmable transport. Biomicrofluidics 7, 014107 (2013).

16. Schilling, K.M., Lepore, A.L., Kurian, J.A. \& Martinez, A.W. Fully enclosed microfluidic paper-based analytical devices. Anal. Chem. 84, 1579-1585 (2012).

17. Martinez, A.W., Phillips, S.T. \& Whitesides, G.M. Three-dimensional microfluidic devices fabricated in layered paper and tape. Proc. Natl. Acad. Sci. U.S.A. 105, 19606-19611 (2008).

18. Cassano, C.L. \& Fan, Z.H. Laminated paper-based analytical devices (LPAD): Fabrication, characterization, and assays. Microfluid. Nanofluid. 15, 173-181 (2013).

19. Fenton, E.M., Mascarenas, M.R., Lopez, G.P. \& Sibbett, S.S. Multiplex lateral-flow test strips fabricated by two-dimensional shaping. ACS Appl. Mat. Inter. 1, 124-129 (2008).

20. Fu, E., Lutz, B., Kauffman, P. \& Yager, P. Controlled reagent transport in disposable 2D paper networks. Lab Chip 10, 918-920 (2010).

21. Lutz, B. et al. Dissolvable fluidic time delays for programming multi-step assays in instrument-free paper diagnostics. Lab Chip 13, 2840-2847 (2013).

22. Houghtaling, J., Liang, T., Thiessen, G. \& Fu, E. Dissolvable bridges for manipulating fluid volumes in paper networks. Anal. Chem. 85, 11201-11204 (2013).

23. Gorkin, R. III et al. Centrifugo-pneumatic valving utilizing dissolvable films. Lab Chip 12, 2894-2902 (2012).

24. Lenk, G.A., Stemme, G. \& Roxhed, N. Delay valving in capillary driven devices based on dissolvable thin films. In 18th International Conference on Miniaturized Systems for Chemistry and Life Sciences, MicroTAS 2014, pp. 216-218 (2014).

25. Lenk, G. et al. A disposable sampling device to collect volume-measured DBS directly from a fingerprick onto DBS paper. Bioanalysis 7, 2085-2094 (2015). 


\section{SUPPLEMENTARY MATERIALS AND METHODS}

\section{Materials and equipment}

Whatman \#1 chromatography paper (Model \#3001-845, Whatman)

Blue food coloring (Acid Blue 9, Great Value Assorted Food Coloring)

Thermal laminating pouches (Model \#TP3854-100, Scotch)

Double sided adhesives (Model \#9495B and Model \#9009, 3M)

Carrier sheets (Model \#4997, 3M)

Polyvinyl alcohol (Model \#360627-25G, Sigma-Aldrich)

Sol-U-Film (Model \#551, Pellon)

Microbore tubing (500 $\mu \mathrm{m}$ ID, Tygon ND-100-80)

Scotch tape (Model \#600K6 (19 mm) and 37348X50CL (48 mm), $3 \mathrm{M})$

PVC films (Model \#C05AS, Oregon Lamination Premium)

Laser cutter (Model \#VLS3.60, Universal Laser Systems.)

Knife plotter (Model \#CE6000-40, Graphtec)

Thermal laminating machine (Model \#TL902, Scotch)

Wax-based printer (Model \#ColorQube 8580/DN, Xerox)

\section{Fabrication of the programmable hydraulic battery}

Hydraulic batteries were designed with Adobe Illustrator using the dimensions shown in Supplementary Table 1 for the inlet region, resistive region, and absorbent region. The inlet region was rectangular with a width, $W$, and a length, $L$. The resistive region was rectangular with a width, $W$, and a length, $L$. In the batteries tested in this work, the absorbent region was a circular sector with a radius, $R$, and central angle, $\theta$. While the absorbent regions presented here are all half circles $\left(\theta=180^{\circ}\right)$ of varying $R$ for consistency, they can also be other shapes.

A $1 \mathrm{~mm}$-wide paper strip was positioned $1 \mathrm{~mm}$ away from the peripheral boundary of the contiguous inlet, resistive and absorbent regions and connected with a single $1 \mathrm{~mm}$ wide strut. The strut makes the handling easier during fabrication. The paper strip was used to create an air gap at the peripheral boundary of the hydraulic battery's porous material after fabrication to prevent fluid leaking out of the hydraulic battery. After lamination, each battery had two openings, one on each face of the inlet region. These openings made the batteries stackable. However, if a single battery was used with a microfluidic device, the opening not connected to the outlet of the microfluidic channel was sealed with single-sided tape. A schematic of the set of hydraulic batteries designed for this work is shown in Supplementary Fig. 1.

Hydraulic batteries were fabricated using the method shown in Supplementary Fig. 2. First, a laser cutter was used to cut holes
Supplementary Table 1 Designed dimensions of hydraulic batteries. The hydraulic batteries are named according to the expected volume to be absorbed, $V$, the fluid used, water $=w$, and the expected, steady-state volumetric flow rate, $Q_{\mathrm{on}}$. A pump that is rated to absorb $32 \mu \mathrm{L}$ of water at $1.5 \mu \mathrm{L} / \mathrm{min}$ is named $32 \mathrm{w} 1.5$ ( $W$ : width, $L$ : length, $R$ : radius, $\theta$ : central angle). Using the $W / L$ and $A_{\text {abs }}$ for each pump, the calibration curves were used to predict the $Q_{o n}, T_{\text {on }}$, and $V\left(Q_{o n}\right.$ : steady-state flow rate $T_{\text {on }}$ : time of pumping, $V$ : volume absorbed).

\begin{tabular}{|c|c|c|c|c|c|c|c|c|c|}
\hline \multirow{2}{*}{$\begin{array}{l}\text { Pump } \\
\text { name }\end{array}$} & \multicolumn{2}{|c|}{ Inlet region } & \multicolumn{2}{|c|}{$\begin{array}{l}\text { Resistive } \\
\text { region }\end{array}$} & \multicolumn{2}{|c|}{$\begin{array}{c}\text { Absorbent } \\
\text { region }\end{array}$} & \multicolumn{3}{|c|}{ Predicted performance } \\
\hline & $\begin{array}{c}W \\
(\mathrm{~mm})\end{array}$ & $\begin{array}{c}L \\
(\mathrm{~mm})\end{array}$ & $\begin{array}{c}W \\
(\mathrm{~mm})\end{array}$ & $\begin{array}{c}L \\
(\mathrm{~mm})\end{array}$ & $\begin{array}{c}R \\
(\mathrm{~mm})\end{array}$ & $\begin{array}{c}\theta \\
\left({ }^{\circ}\right)\end{array}$ & $\begin{array}{c}Q_{\text {on }} \\
(\mu \mathrm{L} / \mathrm{min})\end{array}$ & $\begin{array}{c}T_{\text {on }} \\
(\mathrm{min})\end{array}$ & $\begin{array}{c}V \\
(\mu \mathrm{L})\end{array}$ \\
\hline $32 \mathrm{w} 7.3$ & 6 & 6 & 4 & 4 & 13.5 & 180 & 7.3 & 4.4 & 32.4 \\
\hline 32 w3.5 & 6 & 6 & 2 & 4 & 13.5 & 180 & 3.5 & 9.4 & 32.4 \\
\hline $32 \mathrm{w} 1.5$ & 6 & 6 & 1 & 4 & 13.5 & 180 & 1.5 & 21.1 & 32.4 \\
\hline $32 \mathrm{w} 0.8$ & 6 & 6 & 1 & 8 & 13.5 & 180 & 0.8 & 41.2 & 32.4 \\
\hline $24 w 3.5$ & 6 & 6 & 2 & 4 & 11.7 & 180 & 3.5 & 7.0 & 24.2 \\
\hline $24 \mathrm{w} 1.5$ & 6 & 6 & 1 & 4 & 11.7 & 180 & 1.5 & 15.8 & 24.2 \\
\hline $24 \mathrm{w} 0.8$ & 6 & 6 & 1 & 8 & 11.7 & 180 & 0.8 & 30.8 & 24.2 \\
\hline $16 \mathrm{w} 3.5$ & 6 & 6 & 2 & 4 & 9.5 & 180 & 3.5 & 4.6 & 15.8 \\
\hline $16 \mathrm{w} 1.5$ & 6 & 6 & 1 & 4 & 9.5 & 180 & 1.5 & 10.3 & 15.8 \\
\hline $16 \mathrm{w} 0.8$ & 6 & 6 & 1 & 8 & 9.5 & 180 & 0.8 & 20.2 & 15.8 \\
\hline 8 w3.5 & 6 & 6 & 2 & 4 & 6.7 & 180 & 3.5 & 2.2 & 7.7 \\
\hline $8 w 1.5$ & 6 & 6 & 1 & 4 & 6.7 & 180 & 1.5 & 5.0 & 7.7 \\
\hline $8 \mathrm{w} 0.8$ & 6 & 6 & 1 & 8 & 6.7 & 180 & 0.8 & 9.8 & 7.7 \\
\hline
\end{tabular}

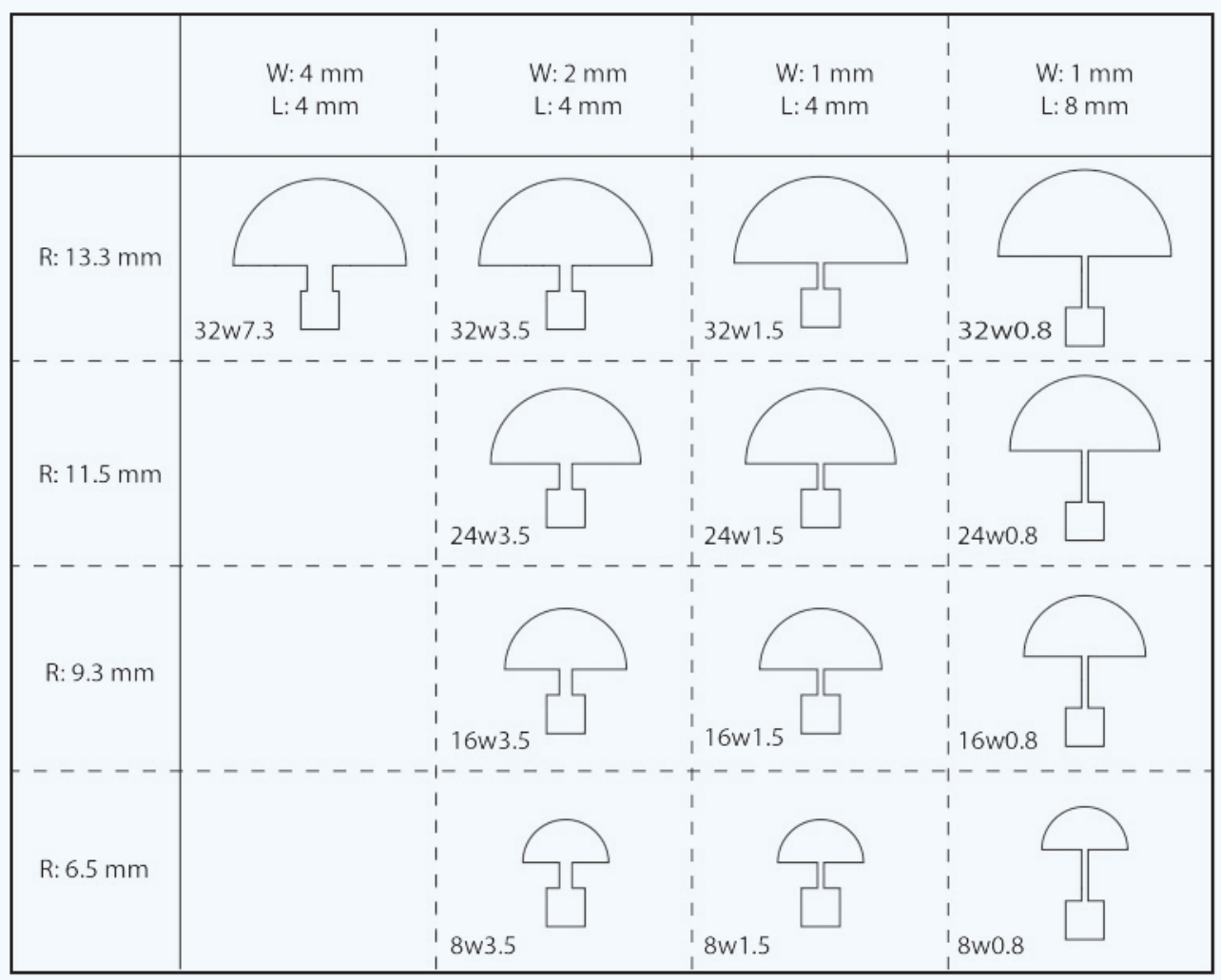

Supplementary Figure 1 Range of hydraulic batteries designed. The name of the hydraulic battery is in the bottom left of each box (W: width, L: length, R: radius). 


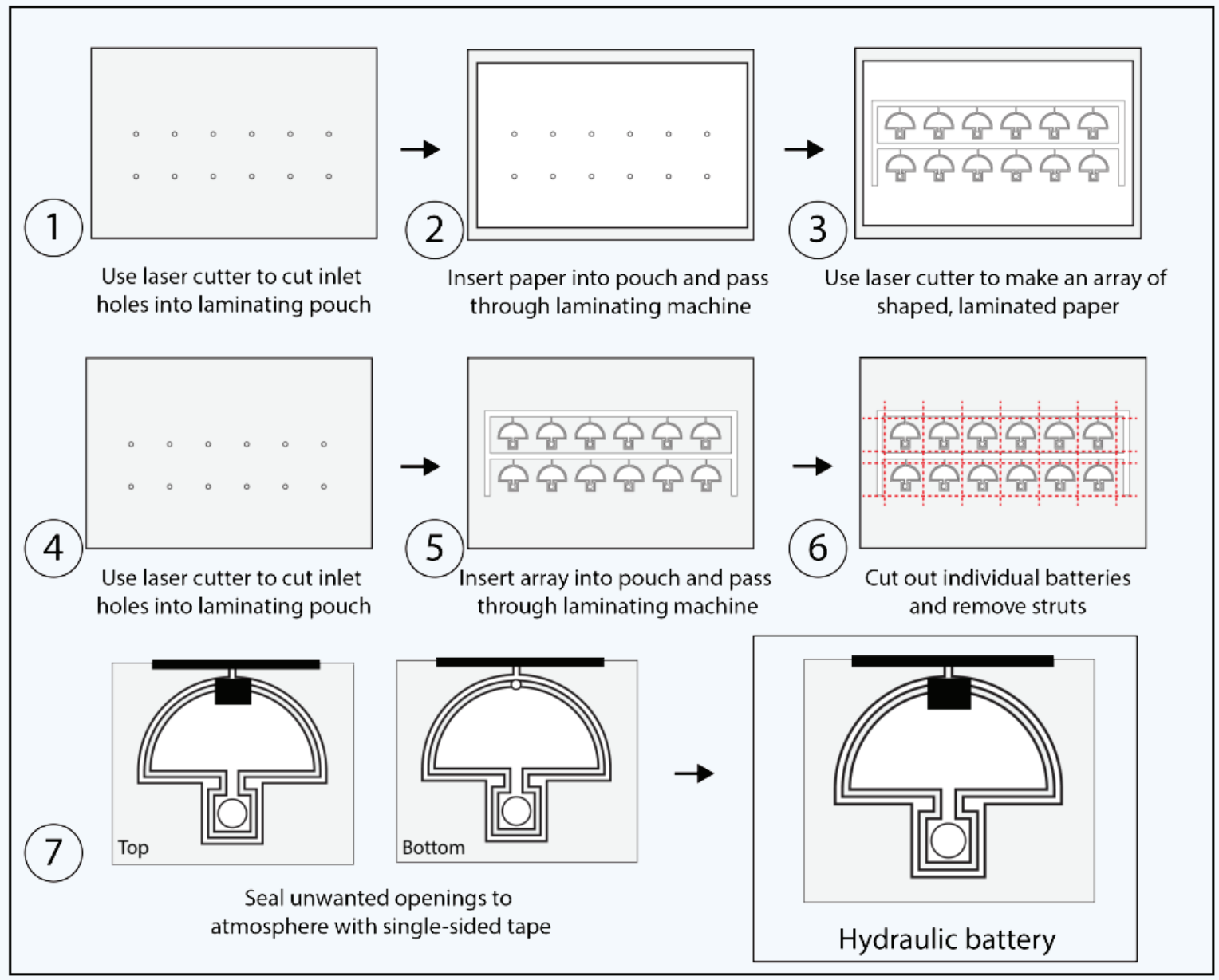

Supplementary Figure 2 Fabrication of hydraulic batteries. The fabrication of hydraulic batteries consisted of a series of laser-cutting and laminating steps.

in thermal laminating pouches that would serve as the openings to the porous material in the inlet region of the hydraulic battery (termed modified pouch). A sheet of Whatman \#1 chromatography paper was then placed in a modified pouch, and this assembly was passed through the thermal laminating machine. Arrays of hydraulic batteries were cut out of this laminated sheet with a laser cutter. This array was then placed in another modified pouch where the inlet holes of the modified pouch were aligned with the inlet holes on the array. This assembly was passed through the thermal laminating machine. Individual batteries were cut out of the array with scissors. The struts were removed with a hole punch $(1.59 \mathrm{~mm})$, leaving a small vent that was connected to the air gap. Air can escape via the vent hole as liquid flows into the hydraulic battery. Each battery, therefore, had 5 layers: laminating film, laminating film, porous material, laminating film, and laminating film. Each laminating film was $76 \mu \mathrm{m}$ thick. Single-sided Scotch tape was used to seal unwanted openings.

The settings for the laser cutter were adjusted to minimize charring of the chromatography paper. The settings used to cut the holes into the laminating pouch were: $6 \%$ power, $25 \%$ speed, $1000 \mathrm{PPI}$, and $0.4 \mathrm{Z}$-axis. The settings used to cut the designs out of the laminated chromatography paper were: $30 \%$ power, $35 \%$ speed, $1000 \mathrm{PPI}$, and $0.4 \mathrm{Z}$-axis. The actual dimensions of the laser-cut material were determined by measuring with an optical microscope. It was determined that when using these settings and hairline-thick lines in the design, the width of a laser-cut line was $200 \mu \mathrm{m}$. The fabricated dimensions of the hydraulic battery are shown in Supplementary Table 2. Dimensions reported in the article are from the fabricated $W / L$ and $A_{\text {abs. }}$.

\section{Fabrication of the stacks of hydraulic battery}

Hydraulic batteries were stacked to generate complex profiles in the microfluidic channel. The inlet regions of each battery in the stack were overlaid, and rings of double-sided adhesive ( $50 \mu \mathrm{m}$ thick) were used to connect adjacent batteries. Disks of porous material ( $3 \mathrm{~mm}$ OD, Whatman \#1 chromatography paper) were inserted within the rings and between the inlet regions to enable fluid transport from the porous material of one battery to that of the next.

Stacks of identical batteries (as shown in Fig. 3b)

$\mathbf{N}=1$ : 32w1.5

$\mathrm{N}=2: 32 \mathrm{w} 1.5$ and $32 \mathrm{w} 1.5$

$\mathbf{N}=3$ : 32w1.5, 32w1.5 and 32w1.5

Stacks generating step-down flow profiles (as shown in Fig. 3c)

SD1: $32 \mathrm{w} 7.3$ and $32 \mathrm{w} 1.5$

SD2: $32 \mathrm{w} 3.5$ and $32 \mathrm{w} 0.8$

Stacks generating decreasing ramp profiles (as shown in Fig. 3d)

R1: 8w3.5, 16w3.5, 24w3.5, and 32w3.5

R2: 8w1.5, 16w1.5, 24w1.5, and 32w1.5

R3: 8w0.8, 16w0.8, 24w0.8, and 32w0.8 
Supplementary Table 2 Actual dimensions of hydraulic batteries ( $W$ : width, $L$ : length, $R$ : radius, $\theta$ : central angle, $W / L$ : width-to-length ratio of the resistive region, $A_{\mathrm{abs}}=$ area of the absorbent region).

\begin{tabular}{|c|c|c|c|c|c|c|c|c|}
\hline \multirow{2}{*}{$\begin{array}{c}\text { Pump } \\
\text { name }\end{array}$} & \multicolumn{2}{|c|}{ Inlet } & \multicolumn{2}{c|}{ Resistive } & \multicolumn{2}{c|}{ Absorbent } & \multicolumn{2}{c|}{ Key variables } \\
\cline { 2 - 9 }$(\mathrm{mm})$ & $\begin{array}{c}W \\
(\mathrm{~mm})\end{array}$ & $\begin{array}{c}W \\
(\mathrm{~mm})\end{array}$ & $\begin{array}{c}L \\
(\mathrm{~mm})\end{array}$ & $\begin{array}{c}R \\
(\mathrm{~mm})\end{array}$ & $\theta\left(^{\circ}\right)$ & $W / L$ & $\begin{array}{c}A_{\mathrm{abs}} \\
\left(\mathrm{mm}^{2}\right)\end{array}$ \\
\hline 32w7.3 & 5.9 & 5.9 & 3.8 & 4.2 & 13.3 & 180 & 0.90 & 278 \\
\hline 32w3.5 & 5.9 & 5.9 & 1.8 & 4.2 & 13.3 & 180 & 0.43 & 278 \\
\hline 32w1.5 & 5.9 & 5.9 & 0.8 & 4.2 & 13.3 & 180 & 0.19 & 278 \\
\hline 32w0.8 & 5.9 & 5.9 & 0.8 & 8.2 & 13.3 & 180 & 0.10 & 278 \\
\hline $24 \mathrm{w} 3.5$ & 5.9 & 5.9 & 1.8 & 4.2 & 11.5 & 180 & 0.43 & 208 \\
\hline $24 \mathrm{w} 1.5$ & 5.9 & 5.9 & 0.8 & 4.2 & 11.5 & 180 & 0.19 & 208 \\
\hline 24w0.8 & 5.9 & 5.9 & 0.8 & 8.2 & 11.5 & 180 & 0.10 & 208 \\
\hline $16 \mathrm{w} 3.5$ & 5.9 & 5.9 & 1.8 & 4.2 & 9.3 & 180 & 0.43 & 136 \\
\hline $16 \mathrm{w} 1.5$ & 5.9 & 5.9 & 0.8 & 4.2 & 9.3 & 180 & 0.19 & 136 \\
\hline $16 \mathrm{w} 0.8$ & 5.9 & 5.9 & 0.8 & 8.2 & 9.3 & 180 & 0.10 & 136 \\
\hline 8w3.5 & 5.9 & 5.9 & 1.8 & 4.2 & 6.5 & 180 & 0.43 & 66 \\
\hline 8w1.5 & 5.9 & 5.9 & 0.8 & 4.2 & 6.5 & 180 & 0.19 & 66 \\
\hline 8w0.8 & 5.9 & 5.9 & 0.8 & 8.2 & 6.5 & 180 & 0.10 & 66 \\
\hline
\end{tabular}

\section{Fabrication of the polyvinyl alcohol delays}

Time delays were fabricated using dissolvable films of polyvinyl alcohol (PVA), which are commonly used in water-soluble packaging of detergents. Two different PVA films were used: one was made in house (house PVA film) and another was purchased (Pellon PVA film). To make the house PVA film, solutions of PVA (9-10 kDa, $80 \%$ hydrolyzed) in DI water $(10 \% \mathrm{w} / \mathrm{v})$ were first prepared and then volumes of this PVA solution were dispensed in petri dishes and left on a hot plate at $80^{\circ} \mathrm{C}$ until visibly dry. Petri dishes were removed from the heat source and cooled to room temperature. Films were then peeled from the dish. The house PVA film that was tested in this work was measured to be $20 \mu \mathrm{m}$ thick. The Pellon PVA film that was tested in this work was measured to be $15 \mu \mathrm{m}$ thick.

Time delays were made from these PVA films. Briefly, individual strips of single-sided scotch tape were placed on each side of a carrying sheet. A smaller punch $(3 \mathrm{~mm})$ was used to create an array of holes in this composite sheet (tape-carrier-tape). This created arrays in each of the single-sided tapes with aligned holes. The tapes were removed from the carrying material and placed on both sides of a PVA film, aligning the holes. A larger punch $(6.35 \mathrm{~mm})$ was then used to remove individual delays. The center of the second punch $(6.35 \mathrm{~mm})$ was aligned with the center of the original hole $(3 \mathrm{~mm})$. The final delays had rings of tape ( $3 \mathrm{~mm} \mathrm{ID/6.35} \mathrm{mm} \mathrm{OD)} \mathrm{adhered} \mathrm{to} \mathrm{both} \mathrm{faces} \mathrm{of} \mathrm{disks} \mathrm{of} \mathrm{PVA} \mathrm{films}$ (6.35 mm OD). Time delays using the house PVA film and Pellon PVA film are termed house PVA delays and Pellon PVA delays, respectively.

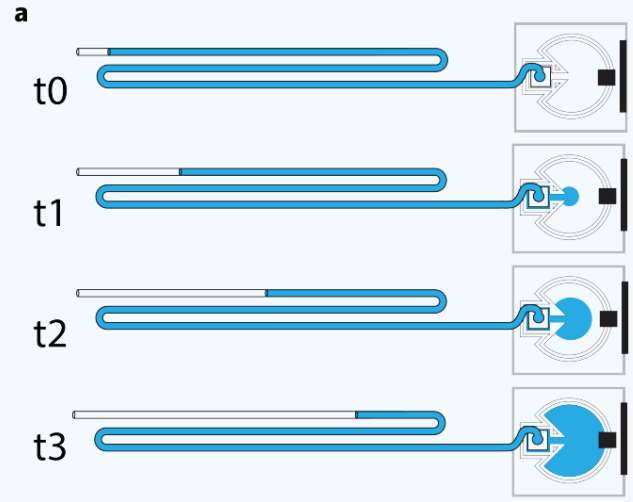

b

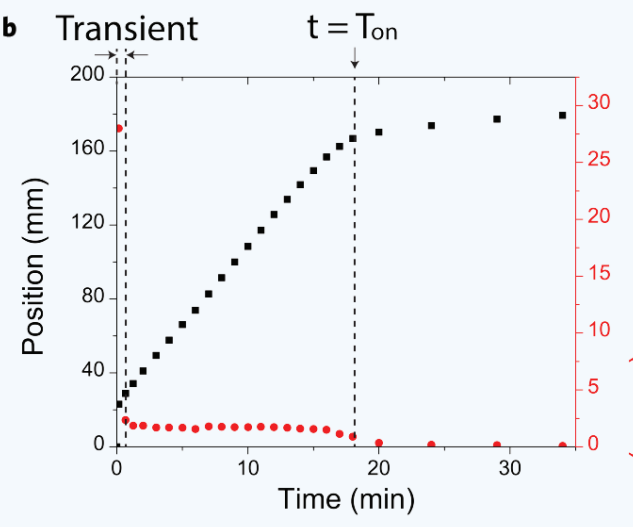

Supplementary Figure 3 Measuring flow-rate profiles. (a) Schematic of how hydraulic batteries were tested using microbore tubing. Upon initial wetting of the inlet region of the hydraulic battery, the position of the lagging front was tracked as a function of time. (b) Exemplary position vs. time data (black squares) and volumetric flow rate vs. time data (red circles) from 32w1.5. During the initial uptake of fluid through the inlet and resistive region, the fluid is pumped at a rate higher than $Q_{o n}$. This transient period lasts approximately 60 seconds for the hydraulic battery tested above and is the time required for the resistive region to be fully wetted. After the transient period, the hydraulic battery pumps fluid at $Q_{\text {on }}$ while $t<T_{\text {on }}$.

\section{Fabrication of the breathable mesh}

Water impermeable meshes were developed for venting of air immediately upstream of the PVA delay. These meshes were created using a modified wax-barrier approach demonstrated in paper microfluidics. Briefly, black, wax-based ink was printed on Whatman \#1 chromatography paper with the ColorQube 8580DN on the highest print quality setting. After printing, this paper was inserted into a $150^{\circ} \mathrm{C}$ oven for 120 seconds to melt the wax and allow it to permeate through the paper. The paper was then removed and allowed to cool. Squares $(10 \mathrm{~mm}$ by $10 \mathrm{~mm}$ ) were cut out of this wax-impregnated paper, and through-holes were cut out of the center of the square with a $3 \mathrm{~mm}$ hole-punch.
To make a linear ramp with $n$-steps that initially pumps at $Q_{\max }$ and decreases at a rate of $-m_{\text {ramp }}$, a stack could be designed as follows. The stack would have $n$ hydraulic batteries, each of which would have a resistive region that pumps fluid into the absorbent region at a flow rate of $Q_{\max } / n$. The hydraulic battery with the smallest volumetric capacity should pump for a given time $\left(T_{1}=Q_{\max } /\right.$ $\left.\left(n \cdot m_{\text {ramp }}\right)\right)$. The remaining hydraulic batteries should have absorbent regions that pull fluid for $2 T_{1}, 3 T_{1} \ldots \mathrm{n} T_{1}$. In this case, the order is fairly unimportant because each battery is effectively connected to the same node. Nonlinear decreasing ramps can be generated by introducing hydraulic batteries into the stack where the steps are not equivalent.

\section{Fabrication of the microfluidic device}

Microfluidic devices were fabricated with by layering solid plastic films and double-sided adhesive. Briefly, rectangular voids $(L: 50 \mathrm{~mm}, W$ : $1 \mathrm{~mm}$ ) were cut into the double-sided adhesive ( $145 \mu \mathrm{m}$ thick) with a knife plotter to serve as the walls of the microfluidic channels. The top and bottom layers of the device comprised PVC films $(125 \mu \mathrm{m}$ thick). The knife plotter was used to size the PVC films ( $L: 75 \mathrm{~mm}, W$ : $25 \mathrm{~mm}$ ) and to cut holes ( $3 \mathrm{~mm}$ ID) into one of the PVC films to serve as inlet and outlet holes into for the microfluidic device. These three pieces were sandwiched together to form a microfluidic device. Rings of double-sided adhesive ( $50 \mu \mathrm{m}$ thick) were used to connect fluid reservoirs to the inlets. 


\section{Fabrication of the stacks of batteries and PVA time delays}

Stacks containing a single hydraulic battery with a single PVA time delay (house PVA delay) were fabricated as follows (Supplementary Fig. 4a). First, a ring of double-sided adhesive $(50 \mu \mathrm{m}$ thick) was placed around the inlet port of the hydraulic battery to be used. Two disks of porous material ( $3 \mathrm{~mm}$ OD, Whatman \#1 chromatography paper) were placed on top of the porous material of the hydraulic battery's inlet region. The PVA delay was then centered on the inlet region, where the face of the PVA delay was in contact with the disks of porous material and the outer edge was in contact with the ring of double-sided adhesive. A larger ring (ID: $6.35 \mathrm{~mm}$ ) of double-sided adhesive ( $145 \mu \mathrm{m}$ thick) was placed on the same level as the PVA delay, with the PVA delay sitting in the opening. Another ring of the double-sided adhesive ( $50 \mu \mathrm{m}$ thick) was placed above this layer. Then, a layer of the breathable mesh was placed with the opening centered on the opening of the double-sided adhesive. On the opposite surface of this breathable mesh, rings of double-sided adhesive (50 $\mu \mathrm{m}$ thick) were attached. This adhesive served to connect the stack to the outlet of the microfluidic device.

The oscillating flow-rate profile was generated by stacking additional elements in a similar manner (Supplementary Fig. 4b). The circular battery was fabricated similarly to the basic programmable battery with the laminating films and paper strip outline. However, instead of having three adjacent sections (inlet region, resistive region, and absorbent region) like the programmable hydraulic battery, the circular battery was a single disk (OD $=20 \mathrm{~mm}$ ) with open ports (inlets) at the center on each face.

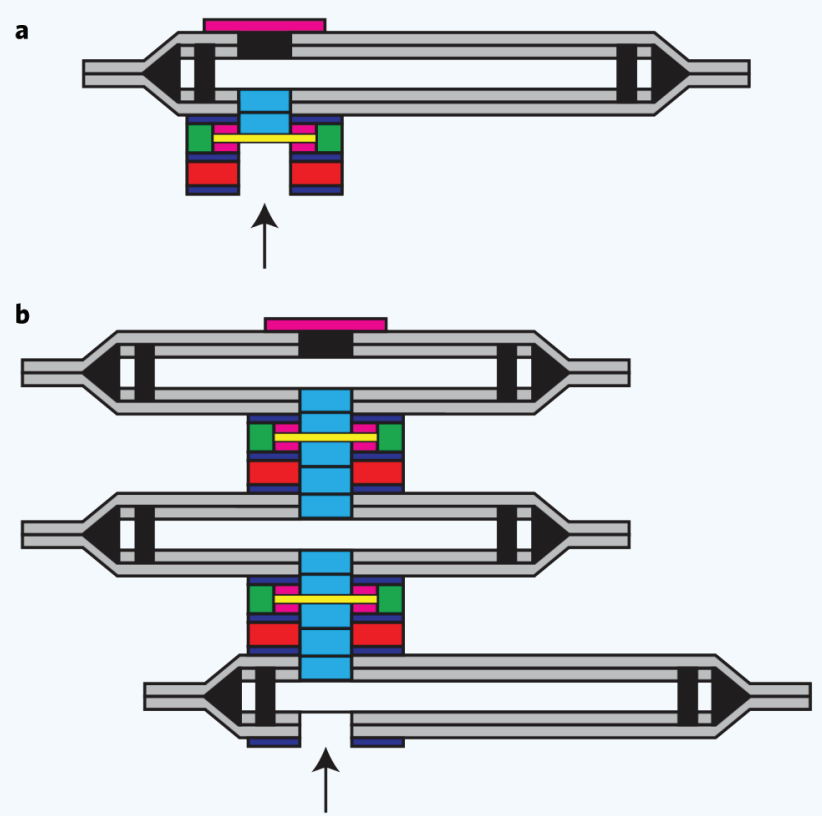

Supplementary Figure 4 Stacks with time delays. (a) Schematic of the stack of a single house PVA delay (20 $\mu \mathrm{m}$ thick) and a single hydraulic battery (32w7.5). (b) Schematic of the stack to generate an oscillating flow rate profile (hydraulic battery (32w0.8), Pellon PVA delay $(15 \mu \mathrm{m}$ thick), circular battery (OD $=20 \mathrm{~mm})$, Pellon PVA delay ( $15 \mu \mathrm{m}$ thick), and circular battery $(\mathrm{OD}=20 \mathrm{~mm})$ ). Arrows display where the microfluidic channel was attached (white: porous material within hydraulic battery, gray: laminating film, yellow: dissolvable PVA, cyan: disks of porous material, magenta: single-sided Scotch tape, red: breathable mesh, blue: $50 \mu \mathrm{m}$ double-sided adhesive, green: $145 \mu \mathrm{m}$ double-sided adhesive, black: air).
Starting with the circular battery, a Pellon PVA delay was connected like previously described. However, instead of connecting the exposed ring of double-sided adhesive to the outlet of a microfluidic device, additional elements were added. This was done by first placing two disks of porous material (3 mm OD, Whatman \# 1 chromatography paper) on the face of the exposed Pellon PVA delay. Another circular battery was then placed on the exposed ring of double-sided adhesive, making sure the porous material of its outlet port was in connection with the disks of porous material. Another Pellon PVA delay was inserted into the stack, using the same approach as listed previously. Finally, with a double-sided adhesive facing outward from a breathable mesh, a hydraulic battery (32w0.8) was connected. The porous material of the battery's outlet port was connected to the disks of porous material. The microfluidic channel was connected to the $32 \mathrm{w} 0.8$ Note that dissolved PVA can increase the viscosity of the fluid and might decrease the flow rates in subsequent batteries.

Stack for single time delay (as shown in Fig. 4 b)

House PVA delay, 32w7.5

Stack for oscillating flow (as shown in Fig. 4c)

32w0.8, Pellon PVA delay, circular battery, Pellon PVA delay, circular battery

\section{SUPPLEMENTARY INFORMATION}

\section{Measuring flow rate profiles}

The pumping element that was to be tested (one or more hydraulic batteries that could include one or more time delays) was connected to the outlet of the microfluidic channel with a ring of double-sided adhesive. The port on the element opposite to this connection was covered with single-sided scotch tape.

For the majority of the experiments, microbore tubing served as the microfluidic channel. PDMS slabs were cored, and the end of a $1.2 \mathrm{~m}$ length of tubing $(500 \mu \mathrm{m}$ ID) was force-fit through the bore. Double-sided adhesive connected the outer face of the PDMS slab to the impermeable plastic barrier of the hydraulic battery to be tested, and the tubing was adjusted to assure that it was abutted to the exposed porous material in the inlet region of the hydraulic battery. A solution (blue food coloring diluted $100 \mathrm{X}$ in water) was loaded into the tubing to make a 1-m plug of fluid $(\sim 203 \mu \mathrm{L})$. In this orientation, the leading fluid front was nearest the hydraulic battery and the lagging fluid front was farthest from the hydraulic battery. A slight hydrostatic pressure (200 Pa) was used to drive the fluid plug toward the battery by lowering the connected hydraulic battery and the leading front of the fluid beneath the lagging fluid front. Immediately after the inlet region wetted, a timer was started. For the majority of experiments, the hydrostatic pressure was then removed by returning the hydraulic battery to the same level as the lagging front. The position of the lagging edge of the fluid was measured as a function of time. A schematic of the setup is shown in Supplementary Fig. 3a. An example of the position vs. time data is shown in Supplementary Fig. 3b.

For experiments that included the dissolvable delay, the open inlet of the stack was connected to the aforementioned microfluidic device. The PDMS slab was connected to the inlet of the microfluidic device, and the $200 \mathrm{~Pa}$ hydrostatic pressure was maintained over the course of the experiment.

The position of the lagging edge as a function of time was then converted into a volumetric flow-rate profile. The cross-sectional area was assumed to be constant and calculated from the reported ID of the tubing $(500 \mu \mathrm{m})$. The change in the position of the lagging front $\left(\Delta X=X_{1}-X_{2}\right)$, where $X_{1}$ and $X_{2}$ are the positions of the front at $t=T_{1}$ and $t=T_{2}$, respectively, was converted into the displaced volume $(\Delta V)$ using the cross-sectional area. Using the associated change in time $(\Delta t=$ $\left.T_{1}-T_{2}\right)$, the volumetric flow rate was then calculated to be $Q=\Delta V / \Delta t$. The 
corresponding flow rates were plotted vs. time to obtain a full flow-rate profile ( $Q$ vs. $t$ ) of a given battery or stack. An example of the volumetric flow rate is shown in Supplementary Figure 3b.

Prior to the complete wetting of the inlet region and the resistive region of the hydraulic battery, the observed flow rates within the channel are initially higher than the steady-state flow rate. Supplementary Fig. $\mathbf{3 b}$ shows an example of a full flow-rate profile. The initial flow rate is transient. However, the flow rate rapidly decreases as the wetted front in the battery moves through the resistive region and becomes approximately constant once the wetted front reaches the absorbent region. Therefore, the time from the initial wetting of the inlet region to the steady-state flow rate can be minimized by minimizing the area of the inlet and resistive regions. Because the majority of the hydraulic battery's void volume is in the absorbent region and the flow rate is limited by the resistive region, the basic hydraulic battery will continue to pump at the approximately constant flow rate for the majority of pumping time.

\section{Fitting data to obtain $\boldsymbol{Q}_{\text {on }}$ and $\boldsymbol{T}_{\text {on }}$}

For each hydraulic battery, the experimentally determined flow-rate profile (like that shown in Supplementary Fig. 3b) was fit with Equation (1) to determine the performance ratings for that given battery $\left(Q_{\mathrm{on}}\right.$ and $\left.T_{\mathrm{on}}\right) \cdot Q_{\mathrm{on}}$ and $T_{\mathrm{on}}$ are the steady-state flow rate and the time of pumping, respectively. The transient period (deemed the first 60 seconds of the flow-rate profile for all batteries) was not included in the fit. The fit minimized its least-squares error to predict the experimental data. Supplementary Table 3 shows the $Q_{\mathrm{on}}, T_{\mathrm{on}}$, and $c$ for the best fits of Equation (1) to the performance of the fabricated hydraulic batteries. The total volume absorbed, $V=Q_{\mathrm{on}} \cdot T_{\mathrm{on}}$ and the respective $\mathrm{R}^{2}$ values (coefficient of determination) are also shown.

As the absorbent region nears saturation, the pump does not immediately switch from pumping at $Q=Q_{\text {on }}$ to $Q=0$. Rather, the flow decays near the end of the pumping time, and that decay is a function of the pore size distribution of the porous material, the area of the absorbent region, and the $W / L$ ratio. In Equation (1), $c$ is a time constant that accounts for this sloped decay.

\section{Generating calibration curves}

The $Q_{\mathrm{on}}$ and the $V$ of a hydraulic battery are determined by its geometry. The $Q_{\mathrm{on}}$ is a function of the $W / L$ ratio of the resistive region, and the $V$

Supplementary Table 3 Experimentally measured pump performance extrapolated from best fit. ( $Q_{\text {on }}$ : steady-state flow rate, $T_{\text {on }}$ : time of pumping, $V$ : volume absorbed, $c$ : time constant describing decay from $Q(\mathrm{t})=Q_{\mathrm{on}}$ to $Q(\mathrm{t})=0 \mu \mathrm{L} / \mathrm{min}, \mathrm{R}^{2}$ : coefficient of determination of the fit).

\begin{tabular}{|c|c|c|c|c|c|}
\hline \multirow{2}{*}{$\begin{array}{c}\text { Pump } \\
\text { name }\end{array}$} & $Q_{\text {on }}$ & $T_{\text {on }}$ & $V$ & \multirow{2}{*}{$c$} & $\mathrm{R}^{2}$ \\
\cline { 2 - 5 } & $(\mu \mathrm{L} / \mathrm{min})$ & $(\min )$ & $(\mu \mathrm{L})$ & $(\min )$ & \\
\hline 32w7.3 & 5.5 & 5.9 & 32 & 0.4 & 0.99 \\
\hline 32w3.5 & 3.7 & 8.5 & 31.6 & 0.7 & 0.98 \\
\hline 32w1.5 & 1.7 & 18.1 & 31.4 & 1.4 & 0.99 \\
\hline 32w0.8 & 0.8 & 44.2 & 35.6 & 4.7 & 0.78 \\
\hline 24w3.5 & 3.5 & 6.4 & 22.3 & 0.3 & 0.94 \\
\hline $24 \mathrm{w} 1.5$ & 1.8 & 12.5 & 22.8 & 0.6 & 0.99 \\
\hline $24 \mathrm{w} 0.8$ & 0.8 & 30 & 23.6 & 2.1 & 0.93 \\
\hline $16 \mathrm{w} 3.5$ & 3.7 & 4.4 & 16 & 0.4 & 0.99 \\
\hline $16 \mathrm{w} 1.5$ & 1.5 & 11 & 16.2 & 0.7 & 0.97 \\
\hline $16 \mathrm{w} 0.8$ & 0.6 & 25 & 16.1 & 1.3 & 0.89 \\
\hline $8 \mathrm{w} 3.5$ & 2.6 & 3.2 & 8.1 & 0.5 & 0.98 \\
\hline $8 \mathrm{w} 1.5$ & 1.9 & 4.9 & 9.3 & 1.2 & 0.99 \\
\hline $8 \mathrm{w} 0.8$ & 1 & 11.2 & 11.2 & 2.5 & 0.97 \\
\hline
\end{tabular}

is a function of $A_{\mathrm{abs}}$. A calibration curve was generated for the material used in this work (laminated Whatman \#1 chromatography paper) to enable the $Q_{\mathrm{on}}$ and $V$ to be predicted for a hydraulic battery with a given $W / L$ ratio and $A_{\mathrm{abs}}$. Simple linear regression was performed using a least squares approach to fit the $Q_{\mathrm{on}}$ vs. $W / L$ data and the $V$ vs. $A_{\text {abs }}$ data. The $y$-intercepts of the fits were set to zero.

There is an expected upper limit to the linearity of $Q_{\mathrm{on}} \mathrm{vs.} W / L$ because the $W / L$ ratio is inversely proportional to the resistance of the resistive region. As explained in the manuscript, the performance of the hydraulic battery is based on the assumption that the resistance of the resistive region is much greater than other regions of the fluidic pathway (e.g., microfluidic channel, inlet region, absorbent region). As the resistance of the resistive region approaches the resistance of other pathways, by increasing the $W / L$, $Q_{\text {on }}$ is no longer linearly dependent on $W / L$. The data from $32 \mathrm{w} 7.3$ was not included in this calibration curve because it falls outside the linear region-the combined resistance of the inlet region and microfluidic channel was calculated to be on the order of the resistive region for 32w7.3.

Assuming the resistive region dominates the fluidic resistance of the system and it is fully wetted, Darcy's Law predicts that the slope of the calibration line for $Q_{\mathrm{on}}$ vs. $W / L$ ( $P_{c} \cdot K \cdot z / \mu$, where $P_{c}, K$, and $z$ are the effective capillary pressure, effective permeability, and thickness of the porous material and $\mu$ is the dynamic viscosity of the fluid. The thickness of Whatman \#1 chromatography paper is $180 \mu \mathrm{m}$, and water has a dynamic viscosity of $0.001 \mathrm{~Pa} \cdot \mathrm{s}$ at room temperature. This indicates that, for Whatman \#1 chromatography paper, $P_{c} \cdot K=7.46^{\star} 10^{-10} \mathrm{~N}$. Because $K$ scales with the effective pore size squared and $P_{c}$ is inversely proportional to the effective pore size, the slope would increase in materials with larger effective pore sizes (for the same solid matrix and fluid). The slope of the calibration line for $V$ vs. $A_{\text {abs }}$ (slope $=0.12 \mu \mathrm{L} / \mathrm{mm}^{2}$ ) is equal to $z \cdot \varphi$, where $\varphi$ is the porosity of the porous material. This indicates that the porosity of Whatman \# 1 chromatography paper is 0.65 .

The calibration curves were then used to predict the $Q_{\text {on }}$ and $V$ for each hydraulic battery (Supplementary Table 1). Hydraulic batteries are named according to the expected volume to be absorbed, $V$, the fluid used, water $=w$, and the expected, steady-state volumetric flow rate, $Q_{\mathrm{on}}$. A pump that is rated to absorb $32 \mu \mathrm{L}$ of water at $1.5 \mu \mathrm{L} / \mathrm{min}$ is named $32 \mathrm{w} 1.5$.

\section{Cost analysis}

The cost analysis of the main two components of a basic hydraulic battery (chromatography paper and laminating pouches) is shown in Supplementary Table 4 . Note that two sheets of Whatman \# 1 chromatography paper ( $300 \mathrm{~mm}$ by $100 \mathrm{~mm}$ ) were used in a single run because they could both fit in a single thermal laminating pouch $(292 \mathrm{~mm}$ by $229 \mathrm{~mm})$. The costs of the double-sided adhesive and single-sided Scotch tape are negligible in comparison.

Supplementary Table 4 Cost analysis of a hydraulic battery.

\begin{tabular}{|c|c|c|c|c|c|c|}
\hline Material & Model \# & $\begin{array}{l}\text { Sheets/ } \\
\text { pkg }\end{array}$ & $\begin{array}{c}\text { Cost/ } \\
\text { pkg }\end{array}$ & $\begin{array}{l}\text { Cost/ } \\
\text { sheet }\end{array}$ & $\begin{array}{l}\text { Sheets/ } \\
\text { run }\end{array}$ & $\begin{array}{c}\text { Cost/ } \\
\text { run }\end{array}$ \\
\hline $\begin{array}{l}\text { Whatman \#1 } \\
\text { chromatography } \\
\text { paper }\end{array}$ & $3001-845$ & 100 & $\$ 65.12$ & $\$ 0.65$ & 2 & $\$ 1.30$ \\
\hline \multirow[t]{4}{*}{$\begin{array}{l}\text { Thermal laminating } \\
\text { pouches }\end{array}$} & ТP3854 & 100 & $\$ 16.98$ & $\$ 0.17$ & 2 & $\$ 0.34$ \\
\hline & & & & & $\begin{array}{l}\text { Total cost } / \\
\text { run }\end{array}$ & $\$ 1.64$ \\
\hline & & & & & $\begin{array}{c}\text { Pumps/ } \\
\text { run }\end{array}$ & 24 \\
\hline & & & & & $\begin{array}{l}\text { Material } \\
\text { cost/ } \\
\text { pump }\end{array}$ & $\$ 0.07$ \\
\hline
\end{tabular}

